\title{
Volumetric Rejuvenation: General Concepts
}

\author{
Samuel M. Lam, MD, FACS ${ }^{1}$ \\ ${ }^{1}$ Willow Bend Wellness Center, Plano, Texas \\ Facial Plast Surg 2015;31:15-21.
}

\begin{abstract}
Address for correspondence Samuel M. Lam, MD, FACS, Willow Bend Wellness Center, 6101 Chapel Hill Blvd., Suite 101, Plano, TX 75093 (e-mail: drlam@lamfacialplastics.com).
\end{abstract}
Abstract
Keywords
- facial volumization
- fat grafting
- fillers
- beauty
- youthfulness
- light and shadow
- facial shape

Volume restoration has become a cornerstone in facial rejuvenation. This article focuses on the aesthetic concepts of volume loss and how to restore volume to mimic nature. The concept of evolving facial shape from a circle, to an upright triangle, to a square, to an inverted triangle is covered. Little model of the inverted egg shape is also proposed. The benefit of restoring an outer facial frame, periorbital frame, and perioral frame is elaborated. Lip volumization is also briefly covered. Finally, understanding the role of highlights and shadows on the impact of aging is addressed.
Today volume restoration has become a cornerstone in the philosophy and execution of surgical and nonsurgical facialrejuvenation strategies for many cosmetic physicians and providers. ${ }^{1-3}$ There are a few dissenters to this paradigm who believe that the face suffers almost exclusively from gravity and from cutaneous aging. However, the model that will be explained herein disagrees with this narrow perspective and espouses that the three methods by which facial aging occurs are volume loss, gravity, and skin changes. A reasonable strategy to overcome these three manifestations of aging should encompass the use of techniques that can address each one of these problems as they are present during the aging process based on a patient's desires, budget, and scope and nature of one's particular aging. This article will set out to describe the philosophy of aging focused on volume loss. The reader is referred to the accompanying chapter "Integrating Injectable Fillers and Fat in Facial Rejuvenation" for details on methods of achieving these goals along with the pros and cons of doing so.

\section{My Philosophy about Aging, Beauty, Cosmetic Procedures, and the Consultation Process}

The goal of any cosmetic endeavor intended for facial rejuvenation should be to achieve patient satisfaction, all the while maintaining the integrity of the physician to achieve natural and safe cosmetic outcomes. Unfortunately, many patients, especially women, come to the physician seeking the correction of very minor cosmetic flaws such as fine perioral rhytids or facial asymmetry that will not improve their appearance in a substantive way even if corrected. I personally believe that it is the duty of the artistic physician to help a patient achieve a better look based on the patient's budget and willingness to accept or decline surgery as an option by suggesting procedures that the patient may not have come to the consultation originally for if it is relevant to the initial complaint of aging. For instance, I believe that hollowing in the temple region is a very crucial component in making a person look older and not so attractive but rarely do I have a patient coming to see me to fill the temple region. Nevertheless, during a consultation in which I believe it is relevant to help restore temple volume loss, I will show before-and-after photographs to help a patient understand how important temple filling can be in making the face look better.

Why not just listen to a patient's desire and go with only that person's requests? Is that not a safer course of action or at least preferred? For me, no. I believe wholeheartedly in suggesting what I believe will help a patient look better for two fundamental reasons. First, many female patients look too closely at the mirror and see things that no one else sees and that I also cannot see. Accordingly, if I cannot see the problem, I rarely can improve the aesthetic deficiency to the patient's satisfaction. As importantly, I believe that
Issue Theme Management of Facial Volume; Guest Editors, Edward D. Buckingham, MD, and Mark Glasgold, MD
Copyright (c) 2015 by Thieme Medical Publishers, Inc., 333 Seventh Avenue, New York, NY 10001, USA. Tel: +1(212) 584-4662.
Dol http://dx.doi.org/ 10.1055/s-0035-1544246. ISSN $0736-6825$. 
performing certain cosmetic procedures even though the patient requests them may simply waste the patient's money and not truly make the person look any better. This is very disturbing to me.

The philosophy described in Malcolm Gladwell's book, Blink, says in short that we make judgments about another person in a nanosecond. Is that person attractive? Is that person youthful? Is that person feminine or masculine? The ultimate goal in my opinion is to help make a person look instantaneously better to another person's perspective not necessarily to oneself (-Fig. 1). Why is that? The reason is that we live in world in which we judge each other all the time, especially this harsh judgment can impact one's social and professional standing. This is particularly true of women who are ruthlessly judged for their beauty and reproductive capacity and that beauty accords them a different status of a mate or position in society. That is oftentimes the unstated reasoning why women or anyone for that matter seeks cosmetic enhancement. Accordingly, my question that I almost always ask a patient when I see a patient after a procedure is "How many compliments have you received?" That is my benchmark for my success. If other people are noticing something unmistakably better about the person I worked on but cannot put one's finger on it, then I believe that I have made a substantive improvement and justified the cost expenditure. In addition, I am also judging my work the instant I lay eyes on a returning patient. Does that person look better in a blink of a second? If so or if not, what else can I do to improve that blink? I constantly look at Facebook or other sources of mother/ daughter photographs to determine what makes a woman look like the mother in this case and not the daughter or vice versa? What shadow is causing one to read the face differently? How about the facial shape? What makes this face attractive to my eye and what does not? I encourage the reader to do the same and to improve one's craft by first improving one's eye to see what defines aging and to give a patient better advice. I use the analogy that if you go to see an attorney, you may have a set of goals but you may not know how to achieve them, so you state to the attorney what your goal is and then permit the attorney to help you navigate the best course of action. This is true for almost all disciplines of a fiduciary nature, and I believe that cosmetic intervention is no different.

Too often, many surgeons speak in the language of their left brain: how many centimeters have I lifted the jawline, how is the reduction in wrinkles in a quantifiable way, etc. The problem with this in my opinion is that many physicians who are not artistic but merely technically gifted can create faces that are less sagging but that either do not look better or are simply unnatural in appearance. What I encourage every cosmetic provider is to really look at the patient as a whole face to see what is truly affecting that person's appearance negatively and when reviewing before-and-after photographs and when looking at a patient postoperatively to see whether that person actually looks better as a whole rather than trying to see if a jawline is cleaner or if a cheek is adequately augmented. In summary, I endeavor to encourage all physicians to truly engage with their craft artistically and to strive to achieve composite results that look more attractive without compartmentalizing the face into regions of improvement. The desire to make an individual look better in a blink of an eye is a principal motivation and passion for me every day when I go to work.

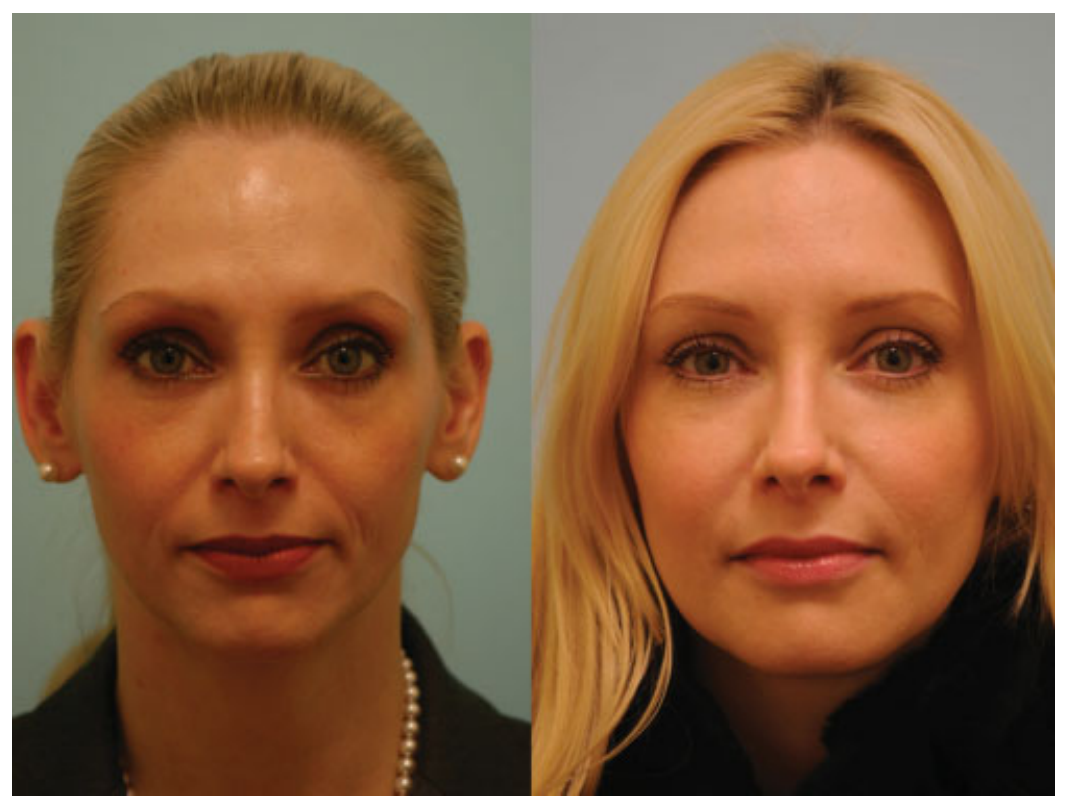

Fig. 1 This patient is shown before (left) at 35 years of age and after (right) at 42 years of age with over 40 syringes of fillers performed over that time. If one uses the concept of blink, to the onlooker, the appearance on the right appears instantaneously much more youthful even though she is in fact 7 years older. Of note, the photograph was taken in the same room, with the same camera, with no ambient light, and with the patient wearing makeup in both images for consistency but the camera picks up much more light on the face in the after photograph. 


\section{Aging Process in Terms of Volume and Facial Shape}

The question may be asked, "What makes a person look unmistakably 20 years old or 40 years old in a blink of an eye to another individual if both of these individuals have no wrinkles or signs of sun damage?" What if there are no obvious signs of gravity such as a sagging brow or a sagging jawline, but you can obviously tell that person is not 20 years old but $40+$ years? The answer lies fundamentally in volume loss, the presence of certain loss of highlights and encroaching presence of senescent shadows, and overall facial shape. These concepts will be more carefully and deliberately elaborated in this section to help a reader understand what makes a face look instantaneously younger or older and thereby how to correct this problem. One caveat should be deposited here: many times the brow region appears to be sagging and the gut instinct of both the surgeon and the patient is to perform a browlift. Curiously, when inspecting photographs of the patient in extreme youth, for example, 20 years old, the brow and eyelid height in many cases has not fallen over time but clearly looks older. The reason for this fact may be attributed to the volumetric collapse of the brow where the underlying bony architecture of the orbital rim is more exposed. The more that a physician looks at old photographs of a patient, the more that he can work to accomplish a result closer to a true youthful state rather than an arbitrary a priori construct of youthfulness that never in fact existed.

The reason that an onlooker can oftentimes tell the age of an individual from across the room even before the minutiae of wrinkles, etc., are visible has a lot to do with overall facial shape and the pattern of highlights and shadows that trigger in our subconscious mind what we consider youthful. First, we will discuss in detail facial shape of youth and then further investigate the concept of highlights and shadows. The loss of volume as one ages is linear in nature from an over exuberance of fat of infancy, a proportionate reduction during childhood, that continues to be lost every year of life until one appears entirely skeletal in old age. The only exception to this model would be the possibility of weight gain as one ages because of poor diet and/or metabolic slowdown. If one controls for weight consistency, then this volume loss is unmistakable, universal, and ineluctable.

To use a reductionist model, I envision the facial shape of a youthful baby, child, and an early adult (early 20s) to be round in nature. The degree of fat that accounts for that roundness would help one to read what age range that person is more precisely (-Fig. 2). As one enters the early 30 s, the ongoing volume loss starts to shape the face to be thinner but interestingly many women actually like this thinning effect because they believe that they look less chubby. If you ask men what they think is more attractive they will instinctually choose a face of a much younger female because they are genetically programmed to mate with a woman of a higher reproductive capacity, that is, younger. As the face progressively loses volume, the underlying skeletal architecture framed at the malar eminence and the chin prominence makes the face more triangular in shape. As the face evolves from the round face of youth to the early aging of the triangle, the face appears to be more of an oval in shape, that is, halfway between a round and a triangle. I believe that this oval shape is very desirable for many women and provides a reasonable end objective for designing a face, which we will talk about more specifically how to do that in the following section. Many women do not desire to be so round in shape, and further I believe that it is hard to achieve that level of youthfulness without the face appearing unnatural and distorted because of small signs of aging that simply cannot be

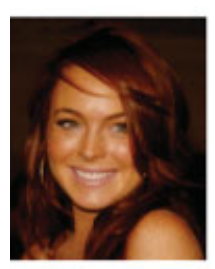

20

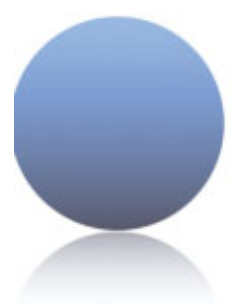

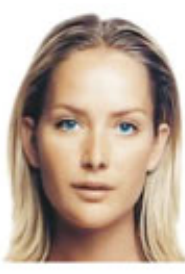

30
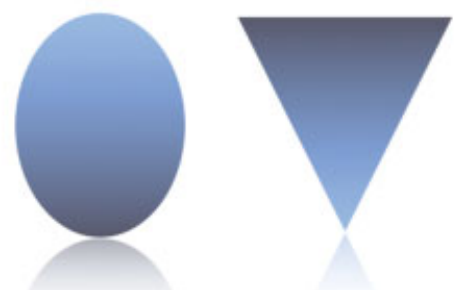

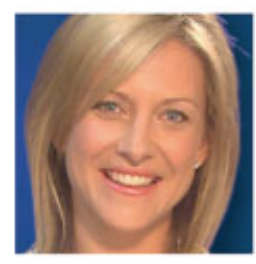

40
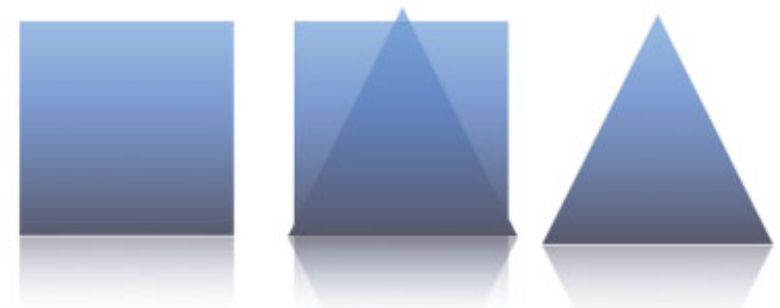

\section{Oval}

Fig. 2 This illustration shows the evolving shape from youth to senescence. We can instantly tell an individual's age based on his or her facial shape. The roundness of youth slowly is lost to reveal the bony skeleton below to form an upright triangle. Ongoing volume loss and early lower facial gravity lead to a squaring off of the face that becomes progressively bottom heavy with an inversion of the initially upright triangle. 
overcome and that would render the face not youthful looking but instead potentially unnatural. As the lower face begins to suffer minor gravitational effects and further loss of volume in one's $40 \mathrm{~s}$, the face starts to become more bottom heavy, which appears to look like a square. This squaring off of the face appears instantaneously more masculine to the onlooker, which robs the face of its former lush femininity. This square progresses to an inverted triangle over time, as the volume loss of the upper face ensues and the gravity of the lower faces continues to dominate. Another way to look at this model is what Dr. William Little describes as an egg with the fat part in the upper half of the face that inverts as one ages (-Fig. 3). Accordingly, the goal with volume restoration is to shape the face in such a way that mimics this appearance.

The center of attention for volume restoration in the past has been the anterior cheek, which adds projection to a flattening face and an extended frame to the eye to render it more youthful. Today, the anterior cheek appears to be overinflated that is particularly unnatural appearing when a person smiles. Instead, I have focused heavily on the outer rim of the face: the temple, the outer zygomatic arch, the area immediately inferior to the zygomatic arch, the outer buccal region, and the prejowl region (-Fig. 4). This outer frame of the face is what gives the face the shape described above of youthfulness. When volume is added to the temple, outer zygomatic arch, area immediately below the zygomatic arch, and the prejowl region, then the face can be designed in such a way as to mimic the egg shape with the fatter side up and the pointier side down. Furthermore, the scalloping that occurs around the outer edges of the face, that is, the alternating concave and convex portions, are changed into a uniform convexity (-Fig. 5). For me, this is why I truly value the frontal view when evaluating my before-and-after results because I can see this change in appearance of the outer face and the impact it has on one's appearance. Furthermore, the frontal view is the view that is most important in establishing one's improved "blink." I refer to this filling the outer edge of the face "facial framing," that is, the perimeter of the face is framed elegantly to make the face appear more youthful. Other methods that can further improve the ideal upright egg-shaped appearance would be a lower face-lift to remove the outer lower facial heaviness associated with an aging face, a chin implant (only if the chin exhibits microgenia) to emphasize the point of the
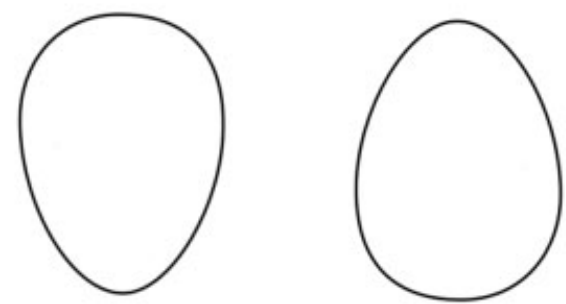

Fig. 3 The face can be modeled as an egg shape as described by Dr. William Little. In youth, the fatter portion of the egg is at the top; and with aging, the egg inverts with dominance present in the region of the lower outer face.

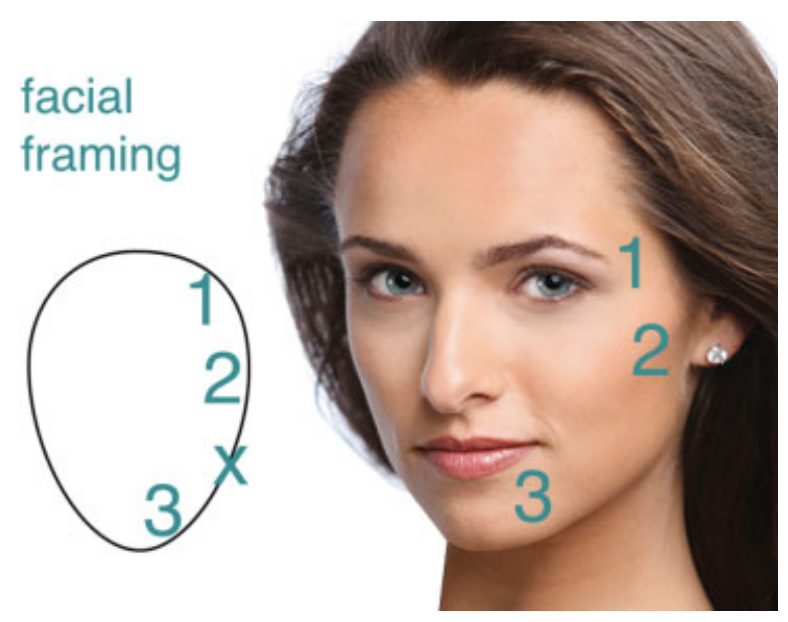

Fig. 4 Creating the egg shape of youth shown in Fig. 3, (1) the areas of concern to augment are the temple, (2) the outer zygomatic arch, the area immediately inferior to the zygomatic arch, the outer buccal region, and (3) the prejowl region.

lower egg, neuromodulator reduction of a bulging masseter (as cosmetically indicated), and hair restoration (to restore the upper facial frame). These adjuncts support the volume reshaping of the face to create the ideal egg shape of the face. The parenthetical remarks above are very important because these adjunctive procedures are only helpful in the right candidate who should benefit from the right procedure. Conversely, I believe that volume restoration is beneficial universally for almost every individual who suffers from mild to significant signs of aging, unless perhaps if the person is grossly obese. Even the mild to moderately overweight person can still benefit from volume restoration because of the illusionary effect that framing the upper face and perioral region can have on making the outer lower face actually appear slimmer in aspect.

While framing the face is a critical aspect in restoring facial volume, I believe that filling around the eyes and around the mouth to frame the eyes and the mouth complete the picture of youthfulness (-Fig. 6). The traditional method of eyelid rejuvenation has been centered on excisional-based surgery, which facetiously has been referred to as blepharectomy rather than blepharoplasty. I believe that in the right patient who has an excess of upper eyelid skin or exuberant fat of the lower eyelid, that a proportional reduction of those areas would be beneficial. However, in almost all the cases some degree of volume restoration in the periorbital region is indicated. In almost every case, I will put some volume along the superior and inferior orbital rim but in selected cases I will also add a conservative skin-only upper blepharoplasty or conservative transconjunctival fat-only lower blepharoplasty. Younger eyes tend to be horizontal in aspect, whereas older eyelids lack this horizontal shape. When tissues are aggressively removed and brows elevated, the natural youthful frame is converted into a more cadaveric appearance that does not mimic youth. Accordingly, the cornerstone in my practice to eyelid rejuvenation is to reestablish the full frame to the eyelid that is present in youth. My colleagues, Mark and 


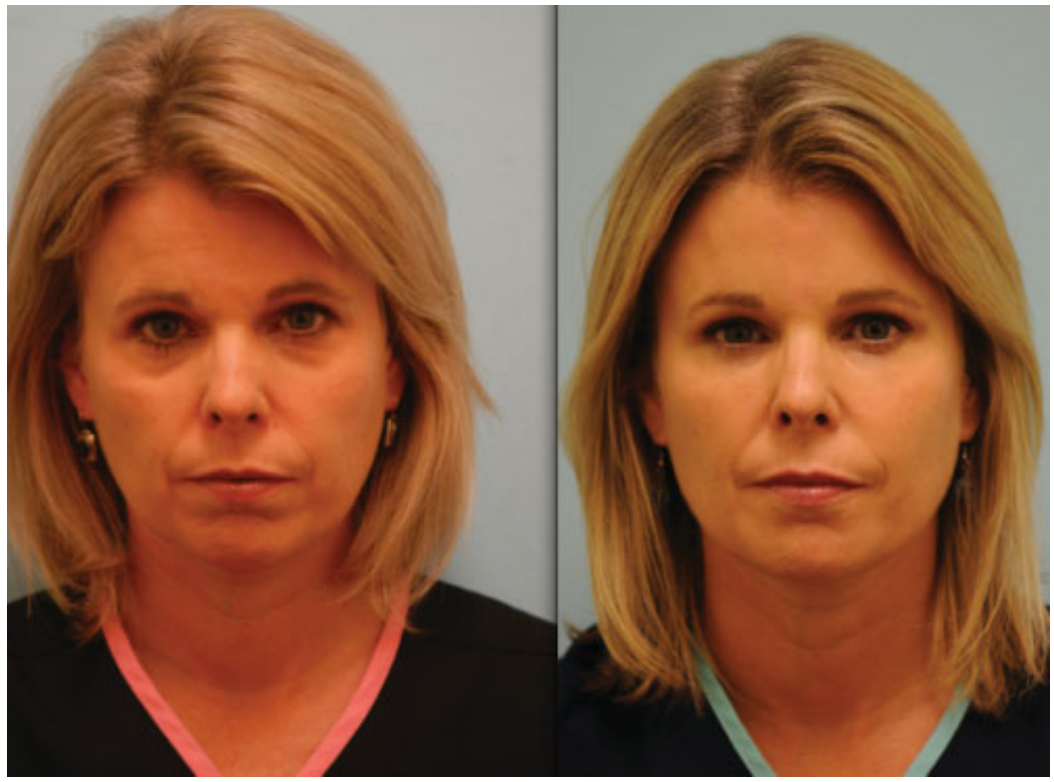

Fig. 5 After facial filling, the face can look much more youthful not only by filling the center of the face but improving the scalloped appearance along the periphery of the face observed from the frontal view.

Robert Glasgold, have correctly identified two types of upper eyelid frames in youth, what they term as type 1 and type 2 , with the former being fuller in aspect and the latter, emptier in aspect. Accordingly, I have become more reliant on evaluating the youthful photograph of a patient for the principal purpose of determining how much upper eyelid framing is needed to recreate the way that the person looked like in his or her own youthful state. The highlight that occurs in the lateral brow aspect is also wonderfully attractive to recreate with volume, a point that will be further discussed later in understanding light and shadow's impact on how we read a face.

I began my career focused on the nasolabial groove as an important area of facial rejuvenation, then I became con-

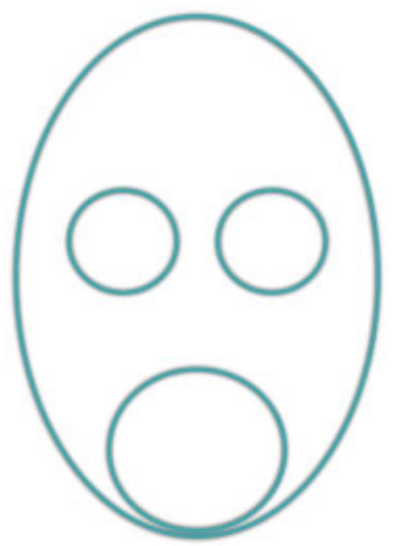

Fig. 6 The three frames that I believe make a face look more youthful are the outer facial frame, the periorbital frame, and the perioral frame. The physician who desires rejuvenating the face can focus his or her attention on these critical elements when designing the face. vinced that filling it was a waste of time as it would not impact the face at all for facial rejuvenation. Today, I stand somewhere in the middle of these two extreme perspectives. I have seen notable improvement in the countenance of my patients who undergo filling in the nasolabial depression but it is still of lesser importance to me than other strategic portions of the face. Although women are obsessed with mouth aging as what they envision as the major reason for their aged appearance, I try to focus them on periorbital aging as most people interact socially and professionally with another person's eyes and not their face. Nevertheless, I believe that oftentimes what ages the mouth is not the mouth but the area that circumscribes the mouth. As mentioned, I look at the lost frame around the eyes and the face as parts of the aging face. Similarly, I imagine the perioral region to suffer the same type of loss of frame. Accordingly, I attempt to fill the prejowl, labiomandibular sulcus, anterior chin/mental sulcus, and nasolabial groove to complete the circle of a youthful frame for the mouth.

It is worth mentioning now a few words regarding lip volumization. Personally, I do not volumize the lips until after the other areas of the face have been rejuvenated for two reasons. First, when volumizing lips before other areas have been done, the lips can look unnatural because they do not match the remainder of the face. Simply put, youthful lips do not appear on older faces. Furthermore, to me it is a waste of money when the rest of the face appears old and the lips look youthful, I believe that priority should be placed on the face over the lips. No matter how hard a surgeon tries it is near impossible to make a face appear to be in one's early 20s and in most cases this is irrelevant and undesirable for a patient. However, I believe that very full lips only naturally look good on a face that is in her young 20s. I am even conservative in lip design for a woman in her 


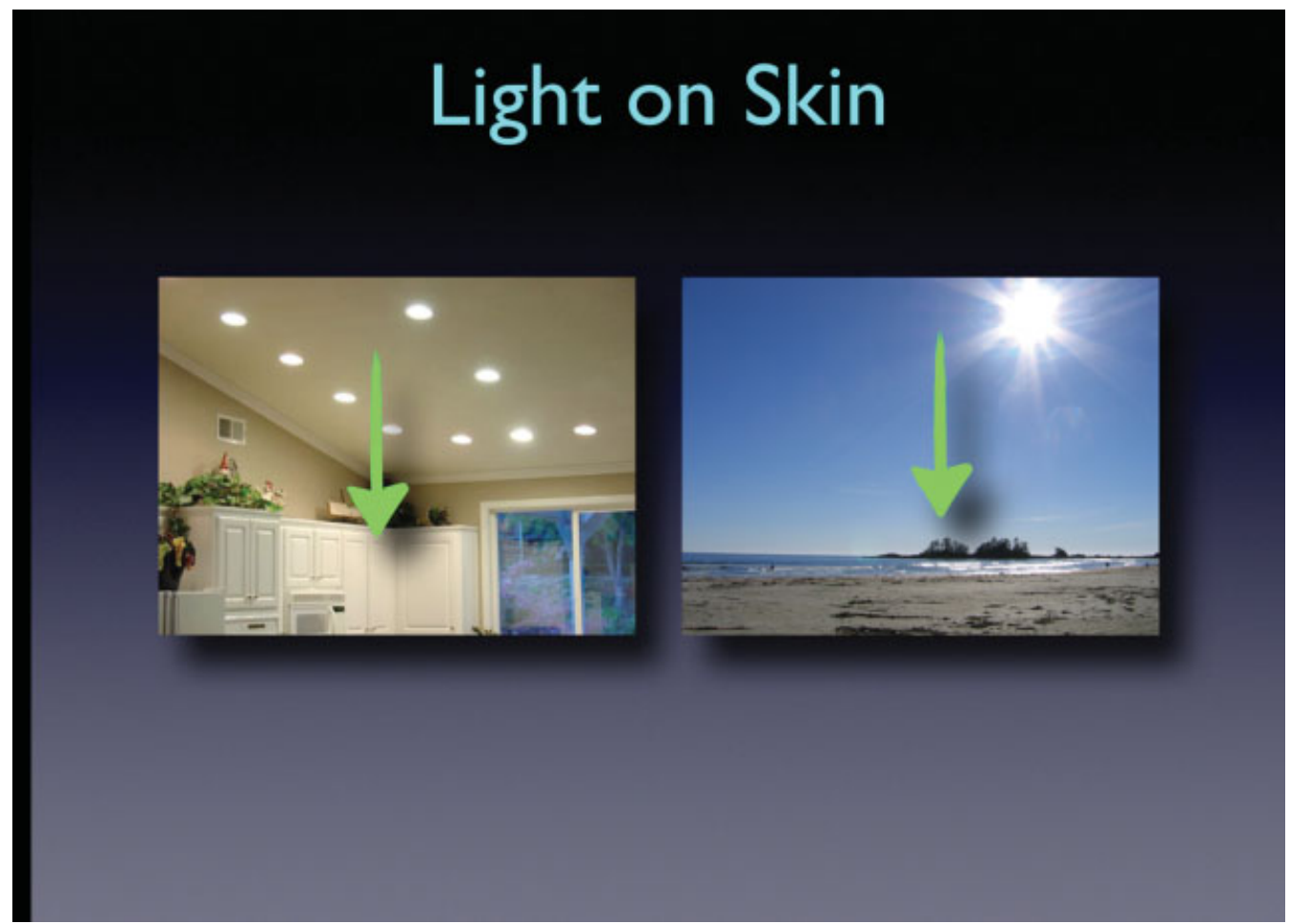

Fig. 7 We live in a world with top-down lighting for the majority of our existence, whether we are indoors or outdoors. When undertaking clinical photography, I do not use flash photography, which I believe fails to mimic nature and also masks the benefits of one's facial volumization results. With top-down lighting being the predominant way we observe each other, recreating the highlights of youth and minimizing the shadows of senescence is an important objective in facial rejuvenation using methods of volumization.

late 20s and early 30s because it simply looks slightly off. Furthermore, I detest borders and almost never fill the vermilion border of the lip, as it simply looks unnatural. I believe that the upper lip should be about a third to slightly less than half of the lower lip with that ratio being on the conservative side depending on how old the person appears in his or her countenance. Also, to make a lip appear natural, it should have a natural taper to no volume laterally and also have shape, that is, points of fullness and emptiness, so that the lip does not look like a sausage. The upper lip should be fuller centrally and just lateral to center to create three mounds that taper to nothing laterally. The lower lip should have two mounds on either side of the midline that also tapers to nothing laterally. With a good aesthetic eye, the surgeon can determine how much lip filler would be appropriate for a patient ( - Fig. 1).

We live in a world with top-down lighting for the majority of our existence, whether we are indoors or outdoors ( - Fig. 7 ). The only exception would be the dimly lit and balanced lighting of a dressing room at a department store to flatter us or with frontal on-camera flash photography. To mimic natural lighting, when I photograph my patients I only use top-down lighting but not so much that extremely harsh shadows are cast. The way to minimize too much shadow overcast involves the right balance of stronger lighting and a smaller photography room. This is also another reason why having a dedicated room for photography is of paramount importance. By replicating how we experience normal lighting, we can achieve results photographically that in my opinion mimic how we see each other. Furthermore, it is important to understand the effect that top-down lighting has on a patient and how we perceive aging in general. The pattern of highlights and shadows that we observe informs our opinion of another person's aging. The more uniform the lighting and the fewer shadow transitions of the face the more youthful the face appears. Furthermore, the highlights of the lateral upper cheek, lateral brow, and anterior chin are hallmarks of youth. Just by replicating certain patterns of light and by diminishing shadows, the surgeon can replicate the semblance of youth. Therefore, when using in-office facial fillers, I believe it is important to have a patient sit upright with a neutral forward gaze to determine the precise requirements of improving highlights and reducing facial shadows. During my off time when I am perusing social media, I often stop to look at a mother-daughter photograph to evaluate what makes the daughter look like the daughter and the mother look like the mother. When we commit our lives to constantly refine our craft, we can achieve increasingly better results, and a large part of that craft must begin with careful, astute observation of nature.

\section{Concluding Thoughts and Summary}

For me, the absolute joy of facial rejuvenation lies in making people look their best, which can change their inner 
confidence and their social/professional standing. This article covered three important concepts to achieve the overall goal of youthfulness: (1) achieving an improved facial shape; (2) framing the face, eyes, and mouth; and (3) improving highlights and diminishing shadows. When physicians begin to explore the artistic side of their mind, they can accomplish work that is balanced, natural, and impactful.

\section{References}

1 Glasgold MJ, Glasgold RA, Lam SM. Volume restoration and facial aesthetics. Facial Plast Surg Clin North Am 2008;16(4):435-442, vi

2 Lam SM, Glasgold RA, Glasgold MJ. Limitations, complications, and long-term sequelae of fat transfer. Facial Plast Surg Clin North Am 2008;16(4):391-399, v

3 Lam SM, Glasgold MJ, Glasgold RA. Complementary Fat Grafting. Philadelphia, PA: Lippincott, Williams \& Wilkins; 2007 